\title{
Dynamic properties of Lennard-Jones fluids and liquid metals
}

\author{
M. Canales \\ Departament de Física i Enginyeria Nuclear, Universitat Politècnica de Catalunya, Campus Nord, Sor Eulàlia d'Anzizu s/n B4-B5, \\ 08034 Barcelona, Spain \\ J. A. Padró \\ Departament de Física Fonamental, Universitat de Barcelona, Diagonal 647, 08028 Barcelona, Spain
}

(Received 5 January 1999)

\begin{abstract}
The dependence of the dynamic properties of liquid metals and Lennard-Jones fluids on the characteristics of the interaction potentials is analyzed. Molecular-dynamics simulations of liquids in analogous conditions but assuming that their particles interact either through a Lennard-Jones or a liquid-metal potential were carried out. The Lennard-Jones potentials were chosen so that both the effective size of the particles and the depth of the potential well were very close to those of the liquid-metal potentials. In order to investigate the extent to which the dynamic properties of liquids depend on the short-range attractive interactions as well as on the softness of the potential cores, molecular-dynamics simulations of the same systems but assuming purely repulsive interactions with the same potential cores were also performed. The study includes both singleparticle dynamic properties, such as the velocity autocorrelation functions, and collective dynamic properties, such as the intermediate scattering functions, the dynamic structure factors, the longitudinal and transverse current correlations, and the transport coefficients. [S1063-651X(99)07707-7]

PACS number(s): 61.20.Lc, 61.20.Ja
\end{abstract}

\section{INTRODUCTION}

Rare gas liquids and liquid metals are two of the most representative families of simple liquids. The interatomic forces for the former are usually modeled by Lennard-Jones (LJ) potentials whereas the situation for the latter is somewhat more complicated because of the presence of the conduction electrons. However, the pseudopotential theory [1] allows us to describe the behavior of ions in liquid metals by considering a simple one-component fluid of electrically neutral pseudoatoms, which interact through an effective liquid metal (LM) potential. The repulsive cores of these effective interactions are softer than those corresponding to the $\mathrm{LJ}$ interactions in rare gas liquids. Moreover, the LM potentials show an oscillatory tail due to the conduction electrons. A deep understanding of the relationship between the properties of different liquids and the characteristics of the corresponding interaction potentials is of great interest to set up the microscopic basis of the liquid-state behavior as well as a useful guide for obtaining more refined potential models. Thus, it would be interesting to relate the most characteristic features of the LM and LJ potentials to the different properties of the corresponding liquids.

Since the pioneering work of Rahman [2] on liquid Rb, the analogies and differences between the properties of liquid metals and those of liquefied inert gases have been the subject of discussion. It is now well known that differences between the structure of these two kinds of liquids are rather small [3]. On the contrary, the differences among the dynamic properties of liquid metals and rare gas liquids are larger and not completely understood. The velocity autocorrelation functions of liquid metals show more pronounced oscillations than those for rare gas liquids [4] and the range of wavelength in which liquid metals and rare gas liquids can support longitudinal and transverse collective modes are markedly different [5]. The differences in the dynamic properties of liquid metals and Lennard-Jones fluids are ordinarily attributed to the different softness of their potential core [6]. However, it has been also suggested that the effects of the attractive forces can be important in certain thermodynamic states $[7,8]$. Besides, the dynamic properties of expanded liquid metals are quite similar to those of LJ fluids [9].

The differences between the properties of liquid metals and rare gas liquids as well as their dependence on the distinctive features of the corresponding interaction potentials were usually based on the results of realistic computer simulations of liquids at different temperatures and densities $[4,6]$. Thus, the discrepancies in the resulting properties cannot be only attributed to the differences in their interaction potentials. In this paper we compare the dynamic properties obtained from realistic simulations of liquid $\mathrm{Li}$ with those for Lennard-Jones fluids at the same thermodynamic conditions (temperature, number density, and atomic mass). Two LM potentials [10-12], which reproduce the most characteristic properties of liquid $\mathrm{Li}$ together with the corresponding $\mathrm{LJ}$ potentials, have been considered. The parameters of the LJ potentials were chosen so that both the effective size of particles and the depth of the potential well were very close to the ones of the corresponding LM potentials. These LJ simulations do not correspond to any realistic liquid but to a liquid model. However, the differences between the properties of these LM and those of the associated LJ systems may be unambiguously attributed to the interaction potentials.

In order to investigate the extent to which the dynamic properties of liquids depend on the attractive interatomic forces, molecular-dynamics (MD) simulations of the same systems but assuming purely repulsive potentials with the same potential core than either the LM or the LJ potentials were also carried out. Moreover, the comparison of the re- 


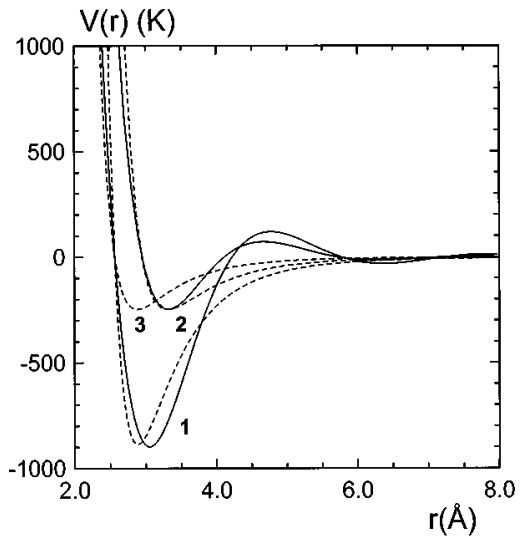

FIG. 1. Interatomic potentials: LM1 and LM2 (solid lines); LJ1, $\mathrm{LJ} 2$, and LJ3 (dashed lines).

sults corresponding to purely repulsive potentials is useful to analyze the influence of the softness of the potential cores on the different properties of the system. The idea of considering the effects of the repulsive and attractive part of the potential is quite old and was already considered in very early theoretical studies of liquids [13]. Because of the cancellations of the attractive interatomic interactions the repulsive forces basically determine the structure of dense nonassociated liquids and perturbation methods such as the WCA theory [14] have been successfully applied to the study of the structure and thermodynamic properties of different systems [15]. However, the influence of the attractive and repulsive interactions on the dynamic properties is little known, especially in the case of collective properties such as those studied in this paper.

\section{COMPUTER SIMULATIONS}

MD simulations of systems made up of 668 particles with the mass of $\mathrm{Li}^{7}$ enclosed in a box with ordinary periodic boundary conditions were carried out. The density and temperature were chosen close to the triple point of $\mathrm{Li}(\rho$ $=4.4512 \times 10^{-2} \AA^{-3} ; T=470 \mathrm{~K}$ ). The properties of the simulated liquids were determined from the configurations generated over runs of about $10^{5}$ time steps. The integration time step was of $3 \mathrm{fs}$. The $k$-dependent properties were calculated for ten different $k$ values between $0.25 \AA^{-1}$ and 4.08 $\AA^{-1}$. The rather low number of particles considered in this study did not allow us to consider values of $k$ smaller than $0.25 \AA^{-1}$. Since MD simulations with low number of particles can produce some spurious results we have performed an extra simulation for the LM1 potential (Fig. 1) using 1750 particles and considering ten $k$ values between $0.185 \AA^{-1}$ and $2.5 \AA^{-1}$. However, we have not observed any significant difference between the MD results from this simulation and those from the simulation with 668 particles.

Two effective LM potentials were considered. The former (LM1), which is based on a pseudopotential with no adjustable parameters, was determined from the atomic number and density of the system by using the neutral pseudoatom method [10]. The second (LM2) is based on the Ashcroft pseudopotential and has an adjustable parameter, the core radius, which was determined by fitting the height of the main peak of the static structure factor to the experimental
TABLE I. Length and energy parameters $(\sigma$ and $\varepsilon)$ of the different potentials. Reduced densities and temperatures $\left(\rho^{*}\right.$ and $T^{*}$ ) corresponding to the systems considered in this paper ( $\rho$ $=4.4512 \times 10^{-2} \AA^{-3}$ and $\left.T=470 \mathrm{~K}\right)$.

\begin{tabular}{lccc}
\hline \hline & $\begin{array}{c}\text { LM1, RLM1 } \\
\text { LJ1, RLJ1 } \\
\text { (state 1) }\end{array}$ & $\begin{array}{c}\text { LM2, RLM2 } \\
\text { LJ2, RLJ2 } \\
\text { (state 2) }\end{array}$ & $\begin{array}{c}\text { LJ3, RLJ3 } \\
\text { (state 3) }\end{array}$ \\
\hline$\sigma(\AA)$ & 2.57 & 2.95 & 2.57 \\
$\varepsilon(\mathrm{K})$ & 887 & 248 & 248 \\
$\rho^{*}$ & 0.756 & 1.143 & 0.756 \\
$T^{*}$ & 0.530 & 1.893 & 1.893 \\
\hline \hline
\end{tabular}

value. More details about these potentials are given in Refs. [10-12]. Despite LM2 showing a first well markedly shallower and located at higher $r$ values than that of LM1 (Fig. 1 ), both the structure and dynamic properties resulting from MD simulations using these two potential models are quite close and show a good accordance with the available experimental data for liquid $\mathrm{Li}[10,11]$. Other effective potential functions recently proposed for liquid $\mathrm{Li}$ [16] are intermediate between LM1 and LM2 but closer to the former. Moreover, when reduced units are used the potential functions for other liquid-alkali metals are intermediate between those for LM1 and LM2 [8,17]. Thus, LM1 and LM2 may be considered as two extreme potential models for simple liquid metals.

We also carried out MD simulations of atoms with the mass of $\mathrm{Li}^{7}$ but assuming $\mathrm{LJ}$ potentials with $\sigma$ and $\varepsilon$ equal to the first zero and to the depth of the first minimum of the LM potentials, respectively. The LJ potentials corresponding to LM1 and LM2 will be termed LJ1 and LJ2, respectively. For the sake of comparison we also performed MD simulations with an intermediate $\mathrm{LJ}$ potential (with $\sigma_{3}=\sigma_{1}$ and $\varepsilon_{3}$ $=\varepsilon_{2}$ ), which will be termed LJ3. The five potential functions are represented in Fig. 1 and the corresponding $\sigma$ and $\varepsilon$ parameters are summarized in Table I. Moreover, we carried out MD simulations of the same systems but truncating the interactions at the position of the first minimum of the potential functions. These five purely repulsive potentials will be termed [repulsive liquid metal (RLM) and repulsive Lennard-Jones (RLJ)] RLM1, RLM2, RLJ1, RLJ2, and RLJ3. It should be pointed out that the cores of the LJ potentials are markedly steeper than the ones of the corresponding LM potentials.

Although the most significant properties of liquid Li assuming either the LM1 or the LM2 potential are similar, the size of particles as well as the relation between the kinetic energy and the depth of the potential well are significantly lower for LM1 than for LM2. Thus, we have determined the states of the different simulated systems in reduced units, according to the usual definitions $\left(\rho^{*}=\rho \sigma^{3}, T^{*}=k_{B} T / \varepsilon\right)$ and using the parameters $\sigma$ and $\varepsilon$ of the LJ potentials. The reduced densities and temperatures of the MD simulations with LM1 and LJ1 are markedly lower than those with LM2 and LJ2, respectively (Table I). So the simulations with these potentials correspond to rather extreme thermodynamic conditions (in reduced units) and they can provide valuable information about the dependence of the results on the effective densities and temperatures. The MD simulations with 
LJ3 are representative of a simple fluid at rather low density and high temperature. We want to emphasize that although all the simulations in this paper were carried out at the same number density and temperature, the differences among the reduced densities and temperatures are large. The states of the different systems may be classified into three groups, which are termed 1, 2, and 3 (Table I).

\section{RESULTS}

\section{A. Single-particle dynamic properties}

The dynamic behavior of single particles in liquids is ordinarily analyzed through the normalized velocity autocorrelation function $C(t)$,

$$
C(t)=\frac{\langle\vec{\nu}(t) \cdot \vec{\nu}(0)\rangle}{\left\langle\nu^{2}(0)\right\rangle} .
$$

The $C(t)$ functions for the systems in this paper show marked differences (for the sake of brevity these functions have not been represented in this paper but they are plotted in Ref. [12]). The $C(t)$ 's for the LM systems present a deep minimum followed by pronounced oscillations whereas for the LJ systems the minimum is shallower and the oscillations are reduced to a shoulder. In the case of LJ3 both the minimum and the shoulder can hardly be observed. The differences between the $C(t)$ functions for LM and LJ liquids should be mainly attributed to the different softness of their potential cores. However, the depth of the minimum and the width of the oscillations/shoulder of the $C(t)$ 's for the purely repulsive potentials are, in general, lower than those for the corresponding full potentials, especially for the systems in the state 1 . This shows that the influence of the attractive forces on $C(t)$ may be important and cannot be generally neglected.

It is very interesting to analyze the motions of single particles in liquids through the spectra of the velocity autocorrelation functions $C(\omega)$. The resulting $C(\omega)$ 's for the systems at state 2 show a broad maximum that reflects the oscillatory motions of the atoms in the cage of their neighbors (Fig. 2). The close agreement between the $C(\omega)$ functions for LM2 and LJ2 and the corresponding ones for RLM2 and RLJ2, respectively, shows that the influence of the attractive forces is almost negligible and the oscillatory atomic motions in very dense liquids are mainly due to close-packing effects. Thus, the differences between the $C(\omega)$ 's for LM2 and LJ2 should be associated with the different softness of the potential cores. However, for the state 1 (at lower density) the influence of the attractive forces on the $C(\omega)$ 's is more significant. So, the peak of $C(\omega)$ for LJ1 is markedly lower than the one for LM1 (the attractive forces are weaker for the former) while for RLM1 and RLJ1 the peak has practically disappeared. These findings indicate that both the close-packing effects, which mainly depend on the characteristics potential cores, and the attractive interatomic interactions contribute significantly to the oscillatory atomic motions in LM1 and LJ1. The results for state 3 are quite different. The $C(\omega)$ functions for LJ3 and RLJ3 are very similar, showing continuous decays without any noticeable maximum. These results reflect that atomic motions in liq-
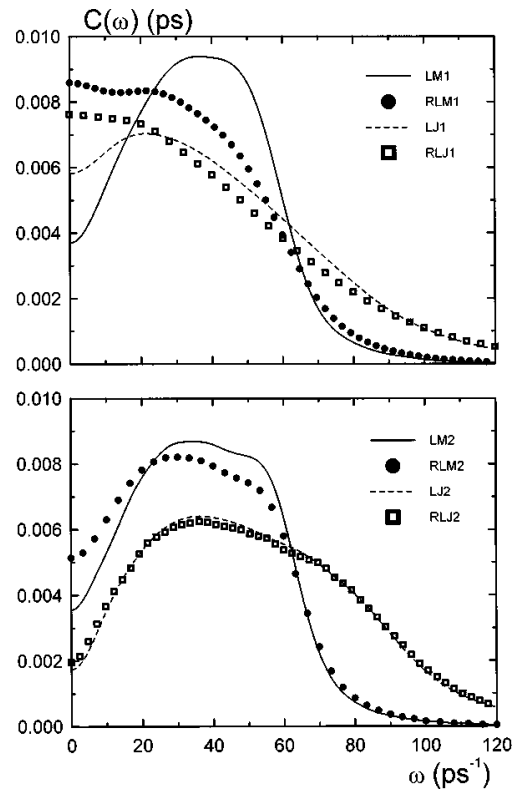

FIG. 2. Spectra of the velocity autocorrelation functions for the systems at the states 1 (up) and 2 (down).

uids at low density and high temperature are mainly diffusive and little influenced by the attractive interatomic interactions.

Unlike for the LJ systems, the $C(\omega)$ spectra for the LM systems show an incipient side peak shifted towards the higher frequencies (Fig. 2). The presence of this side peak, which can also be observed for the RLM potentials, should be related to the softness of the LM potential cores and is consistent with earlier results for soft-sphere liquids [18]. Another difference between the LM and LJ liquids is the range of frequencies in the $C(\omega)$ spectra. $\quad C(\omega)$ for the LM systems is almost zero at frequencies higher than $\omega$ $=100 \mathrm{ps}^{-1}$ whereas for the LJ systems there is a noticeable contribution of these high frequencies (Fig. 2). These results, which are also in accordance with those for soft-sphere liquids [18], suggest that the existence of rather slow collective oscillatory motions in liquids is more difficult when the cores of the interaction potentials are harder. The initial values of $C(\omega)$ reflect the contribution of the purely diffusive motions to the spectrum, being $C(0)$ directly related to the selfdiffusion coefficients.

The self-diffusion coefficients $D$ have been determined by integration of the $C(t)$ function as well as from the long-time slope of the mean-square displacement [3]. The results obtained by the two methods are in good accord and they are gathered in Table II. In all cases, the $D$ coefficients for the full potentials are lower than those for the corresponding repulsive potentials, which corroborates that the diffusive motions are hindered by the attractive interactions. This is more notorious in the case of potentials that produce strong attractive interactions, such as LM1. As may be observed in Table II the values of $D$ for RLM1 and RLM2 are larger than those for RLJ1 and RLJ2, respectively. According to this finding the values of the $D$ coefficients should increase with the softness of the potential cores.

Another property frequently employed to study the motions of atoms in liquids is the self-intermediate scattering function $F_{s}(k, t)[3,19]$, 
TABLE II. Results from the MD simulations according to the definitions given in the text. $D$ $\left(10^{-9} \mathrm{~m}^{2} \mathrm{~s}^{-1}\right), k\left(\AA^{-1}\right), S(0)\left(10^{-2}\right), \eta_{s}\left(10^{-4} \mathrm{Pas}\right), \eta_{l}\left(10^{-4} \mathrm{~Pa} \mathrm{~s}\right)$, and $\lambda\left(\mathrm{J} \mathrm{m}^{-1} \mathrm{~s}^{-1} \mathrm{~K}^{-1}\right)$.

\begin{tabular}{llccccccccc}
\hline \hline & LM1 & RLM1 & LJ1 & RLJ1 & LM2 & RLM2 & LJ2 & RLJ2 & LJ3 & RLJ3 \\
\hline$D$ & 6.6 & 16.0 & 10.0 & 14.0 & 6.3 & 9.0 & 2.8 & 3.4 & 22.6 & 26.0 \\
$k_{L}^{\max }$ & $1.84^{\mathrm{a}}$ & 0.88 & & 0.44 & 1.84 & 1.44 & 1.25 & 1.02 & 0.44 & 0.44 \\
$k_{T}^{\min }$ & 0.25 & 1.02 & 0.88 & 1.25 & 0.25 & 0.44 & 0.25 & 0.25 & & \\
$k_{T}^{\max }$ & 4.08 & 1.84 & 4.08 & 1.44 & 4.08 & 4.08 & 4.08 & 4.08 & & \\
$S(0)$ & 2.5 & 3.0 & & 3.0 & 2.0 & 2.0 & 1.5 & 1.5 & 8.0 & 5.5 \\
$\eta_{s}$ & 5.1 & 1.9 & 3.3 & 2.35 & 5.8 & 3.4 & 12.8 & 11.0 & 1.4 & 1.3 \\
$\eta_{l}$ & 8.5 & 3.2 & 60.5 & 4.4 & 8.6 & 5.6 & 24.3 & 15.1 & 2.4 & 2.3 \\
$\lambda$ & 1.3 & 0.8 & 1.0 & 1.0 & 1.3 & 1.2 & 1.5 & 1.5 & 0.8 & 0.7 \\
\hline
\end{tabular}

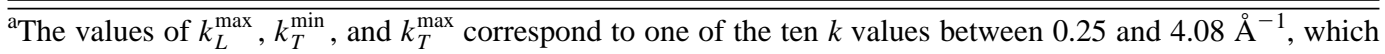
were considered in this study.

$$
F_{s}(k, t)=\langle\exp (-i \vec{k} \cdot[\vec{r}(t)-\vec{r}(0)])\rangle
$$

During the MD simulations we determined these functions for ten values of $k$ and the more representative results are shown in Fig. 3. In the hydrodynamic limit, the $F_{s}(k, t)$ functions should have an exponential behavior $F_{s}(k, t)$ $=\exp \left(-D k^{2} t\right)$, where $D$ is the self-diffusion coefficient. At low $k$ 's, our $F_{s}(k, t)$ functions, for a given $k$, show very slow exponential decays, which are consistent with the hydrodynamic predictions. Moreover, the rate of these decays changes according to the values of the $D$ coefficients. The higher $D$ is, the faster the $F_{s}(k, t)$ decay is. When $k$ increases, the decay of $F_{s}(k, t)$ becomes faster but the qualita-
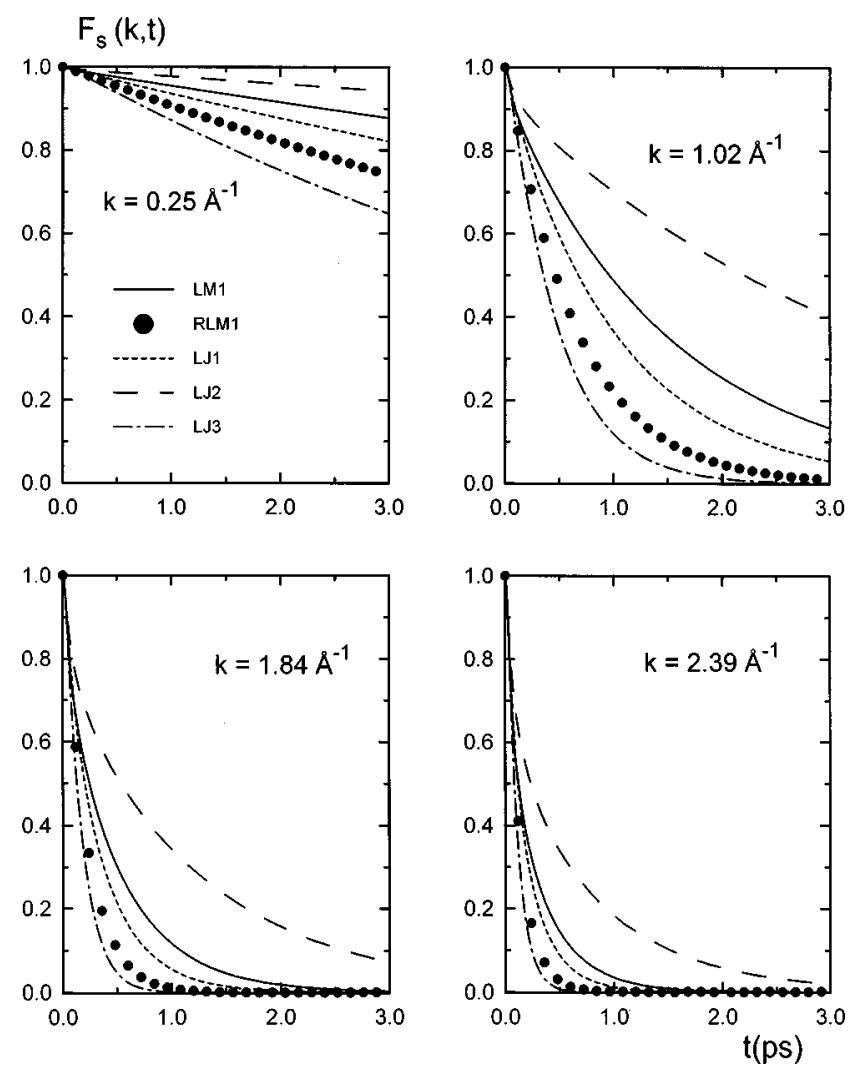

FIG. 3. Normalized intermediate scattering functions at different values of $k$. tive differences between the decay of the functions for the different systems are similar to those observed at low $k$ 's.

\section{B. Dynamic structure factors and longitudinal current correlations}

The intermediate scattering functions $F(k, t)$ are defined as $[3,19]$

$$
F(k, t)=N^{-1}\left\langle\rho_{\mathbf{k}}(t) \cdot \rho_{-\mathbf{k}}(t)\right\rangle,
$$

where

$$
\rho_{\mathbf{k}}(t)=\sum_{j=1}^{N} \exp \left[-i \vec{k} \cdot \vec{r}_{j}(t)\right]
$$

The dynamic structure factors $S(k, \omega)$ (Figs. 4-6) were calculated by Fourier transforming the $F(k, t)$ functions determined during the MD simulations. The $S(k, \omega)$ functions for LJ1 are not included in Fig. 4 because the very slow decays of the $F(k, t)$ functions at low values of $k$ did not allow us to obtain reliable $S(k, \omega)$ results. This anomalous behavior should be associated with the extreme thermodynamic conditions (very low $T^{*}$ and $\rho^{*}$ ) of this liquid model. In the hydrodynamic regime, the $S(k, \omega)$ functions, for a given $k$, should present a Brillouin peak at nonzero frequency that is associated with the propagation of the density fluctuations $[3,19]$. This Brillouin peak should disappear as $k$ increases and the behavior of the system approaches to that characteristic of the kinetic regime. The maximum value of $k$ for which $S(k, \omega)$ shows a noticeable Brillouin peak $\left(k_{L}^{\max }\right)$ was determined for each system and the results are listed in Table II. It should be noted that $k_{L}^{\max }$ could not be accurately determined because $S(k, \omega)$ was only calculated for ten values of $k$. Moreover, the extinction of the Brillouin peaks as $k$ increases is gradual. During the MD simulations we also determined the longitudinal-current correlation functions defined as $[3,19]$

$$
C_{L}(k, t)=\frac{k^{2}}{N}\left\langle j_{\mathbf{k}}^{z}(t) \cdot j_{-\mathbf{k}}^{z}(0)\right\rangle
$$



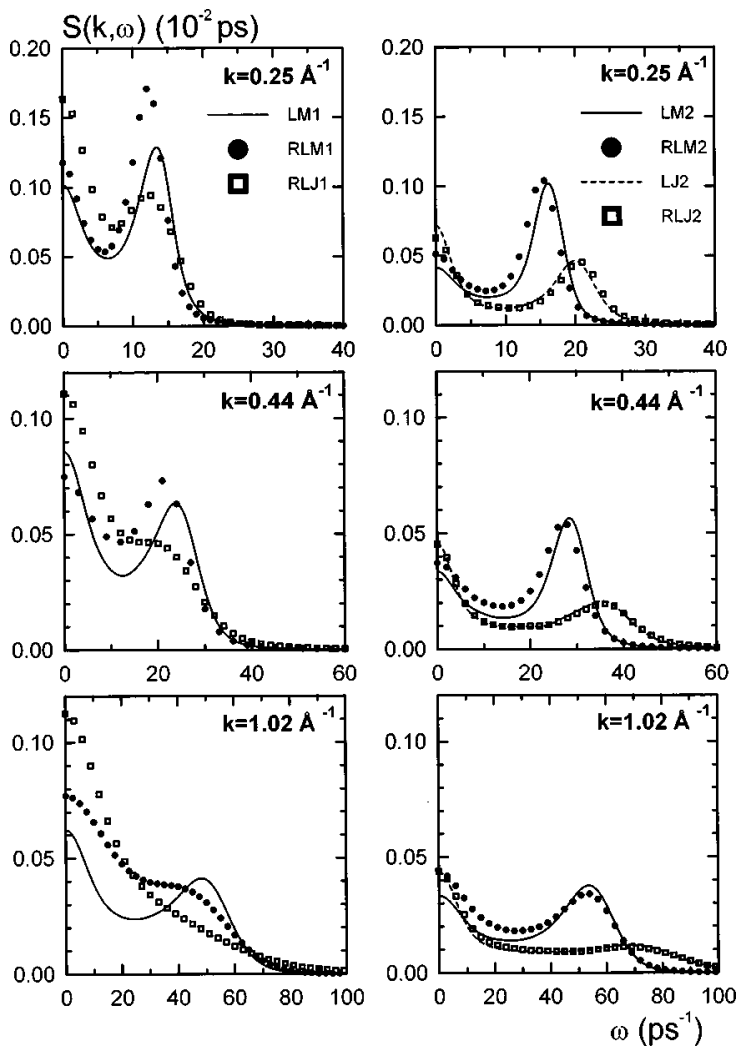

FIG. 4. Dynamic structure factor at low $k$ values for the systems at the states 1 (left) and 2 (right).
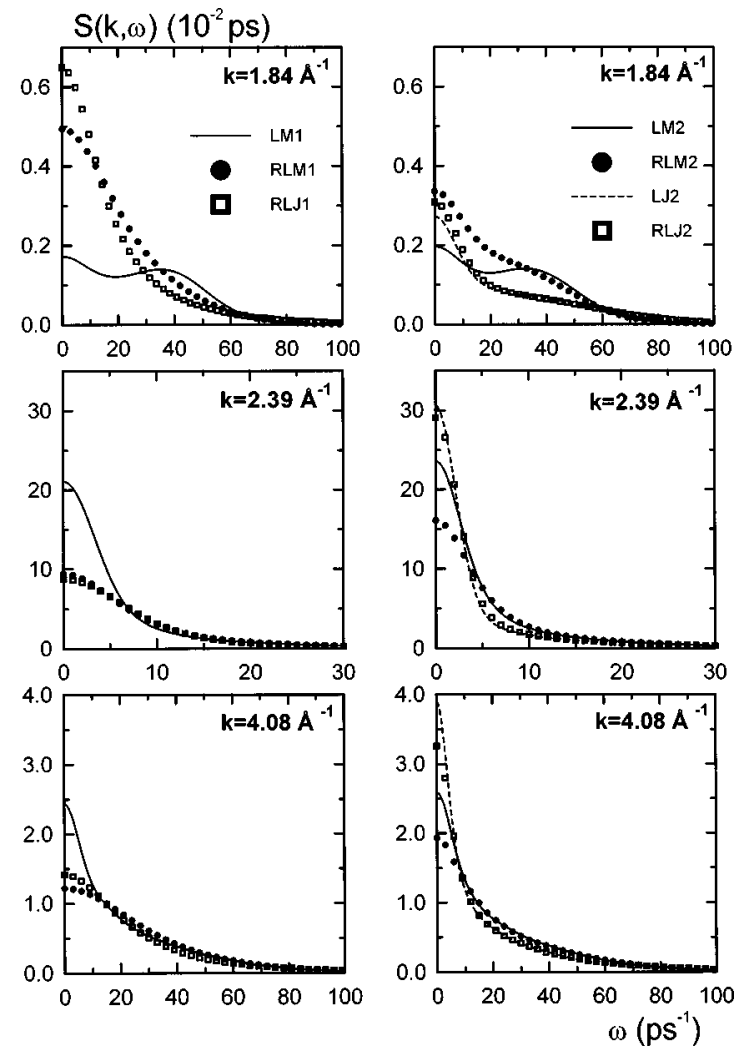

FIG. 5. Dynamic structure factor at high $k$ values for the systems at the states 1 (left) and 2 (right).
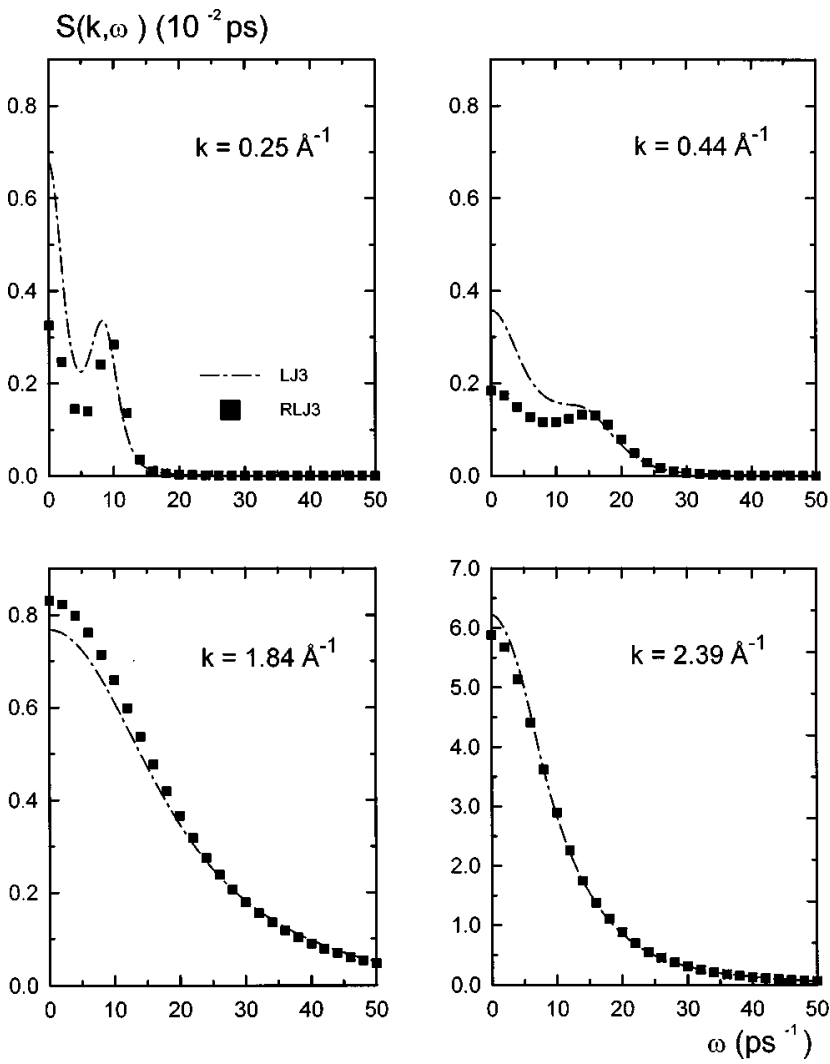

FIG. 6. Dynamic structure factor for the systems at the state 3 .

where $j_{\mathbf{k}}^{z}(t)$ is the part of the current function $\left[\vec{j}_{\mathbf{k}}(t)\right]$ that is parallel to the direction of the wave vector $\vec{k}$. The current function $\vec{j}_{\mathbf{k}}(t)$ is given by

$$
\vec{j}_{\mathbf{k}}(t)=\sum_{l=1}^{N} \vec{\nu}_{l}(t) \cdot \exp \left[-i \vec{k} \cdot \vec{r}_{l}(t)\right] .
$$

The longitudinal current spectra $\left[C_{L}(k, \omega)\right]$ calculated as the Fourier transforms of the $C_{L}(k, t)$ functions are in good agreement with those obtained through the equation $C_{L}(k, \omega)=\omega^{2} S(k, \omega)[3,19]$. The positions of the $C_{L}(k, \omega)$ peaks for each $k$ were used to plot the longitudinal dispersion curves (Fig. 7). These dispersion curves are close to those obtained in the low- $k$ region by visual inspection of the Brillouin peaks of $S(k, \omega)$ [11]. The initial slopes of the dispersion curves are closely related to the velocity of propagation of the density fluctuations (sound waves) in the liquid [19].

The $S(k, \omega)$ results for low values of $k$ corresponding to the states 1 and 2, are shown in Fig. 4. The $S(k, \omega)$ functions for LM2 show higher Brillouin peaks that spread over larger values of $k$ than those for LJ2 (see $k_{L}^{\max }$ in Table II). This suggests that the propagating longitudinal modes are better sustained by liquid metals than by Lennard-Jones fluids. It should be emphasized that the $S(k, \omega)$ functions for the full potentials (LM2 and LJ2) are very close to those for the respective repulsive potentials (RLM2 and RLJ2). This confirms that the marked differences between the results for LM2 and LJ2 should be attributed to the different softness of their potential cores. If the results for RLM1 and RLJ1 are compared, both the higher Brillouin peak (Fig. 4) and the larger $k_{L}^{\max }$ value (Table II) also correspond to the potential 

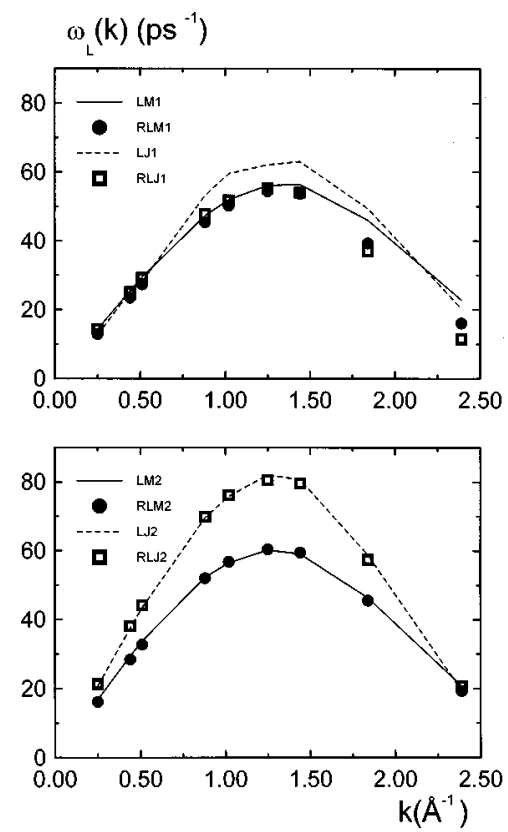

FIG. 7. Longitudinal dispersion curves for the systems at the states 1 (up) and 2 (down).

with the softer core. Our findings corroborate the strong influence of the softness of the potential core on the propagation of longitudinal modes. Nevertheless, our results also suggest that this influence is more marked in liquids at high densities and the effects of the attractive forces become more important as density decreases. This is consistent with the noticeable differences between the $S(k, \omega)$ functions for the LJ3 and RLJ3 potentials at low- $k$ values (Fig. 6).

The Brillouin peaks for the LM2 and RLM2 potentials are located at smaller values of $\omega$ than the ones for the LJ2 and RLJ2 potentials. Thus, the corresponding dispersion curves show noticeable differences (Fig. 7) and the adiabatic sound velocity for the former systems is lower than that for the second. As with $S(k, \omega)$, there are no noticeable effects of the attractive interactions on the dispersion curves. It should be noted that the sound velocity in liquid-alkali metals close to the melting point is higher than that for rare-gas liquids [3], which is in disagreement with our results for state 2. This discrepancy should be attributed to the different thermodynamic state of the compared real systems. It should be emphasized that the results in this paper corroborate that the lower the isothermal compressibility is [see $S(0)$ in Table II], the higher the adiabatic sound velocity is. The results for the state 1 are quite different from those for the state 2 . The Brillouin peaks for LM1, RLM1, and RLJ1 are located at similar positions and the corresponding dispersion curves (Fig. 7) as well as the adiabatic sound velocity show little differences for these systems. According to our results the influence of the softness of the potential core on the propagation of the longitudinal collective modes depends on the thermodynamic state of the system, being more marked at lower packing fractions. It should be pointed out that, in earlier studies of hard-core fluids [20,21], the propagation of collective modes was practically independent of the thermodynamic state.

The influence of the attractive part of the LM potentials on the $S(k, \omega)$ functions at higher wave numbers is more marked (Fig. 5). So, for example, $S(k, \omega)$ at $k=1.84 \AA^{-1}$ shows a clear Brillouin peak for both LM1 and LM2 whereas there is no Brillouin peak for RLM1 and only a reminiscent shoulder for RLM2. The effects of the attractive forces for the LJ potentials are smaller than those for the LM potentials, as corresponds to the weaker strength associated with the attractive parts of the former. This is confirmed by the close agreement between the $S(k, \omega)$ 's for LJ2 and RLJ2. Moreover, the differences between the $S(k, \omega)$ 's for LJ3 and RLJ3 at high $k$ 's are rather small (Fig. 6). On the other hand, it is interesting to point out that the discrepancies between the $S(k, \omega)$ 's for RLM2 and RLJ2 are clearly larger than those between RLM1 and RLJ1. Thus, also for high $k$ 's, the influence of the softness of the potential core on $S(k, \omega)$ becomes weaker as the density decreases.

The static structure factors $[S(k)]$ in the low- $k$ region were determined from the intermediate scattering functions according to the relation $S(k)=F(k, 0)$. The $S(0)$ values, which are proportional to the isothermal compressibility coefficients, were obtained by extrapolating the $S(k)$ functions $[8,11]$. The results are gathered in Table II. $S(0)$ for LJ1 could not be accurately determined since the $S(k)$ function for this system shows a dramatic increase as $k$ tends to zero. This is consistent with the mentioned extremely slow decay of $F(k, t)$ at low $k$ 's. Except for LJ3 and RLJ3, the values of $S(0)$ for the different systems are similar, being the discrepancies of the same order of magnitude than the estimated errors in the determination of $S(0)\left( \pm 5 \times 10^{-3}\right)$. The $S(0)$ results for LM2 and LJ2 suggest that the influence of the attractive interactions is small for systems at high density and temperature. However, it has been observed that both the short-range attractive and the long-range oscillatory forces of the LM1 potential have a large influence on $S(0)$. Since the two contributions are similar but in the opposite direction [8] the $S(0)$ values for LM1 and RLM1 are quite close. As with $S(k, \omega)$, the values of $S(0)$ for systems at low density are significantly influenced by the attractive interatomic forces, which is reflected by the different values of $S(0)$ for LJ3 and RLJ3.

As was already noted in Sec. I, the notorious differences between the dynamical behavior of liquid metals and raregas liquids were associated with the lower compressibility of liquid metals (liquid-alkali metals are significantly less compressible than rare-gas liquids at states close to their melting points). This, in turn, was related to the softer core of the LM potentials $[3,19]$. However, our findings suggest that the correlation between the dynamical properties of a system and its isothermal compressibility is not evident. So, the dynamic structure factors and related properties of the liquids in the states 1 and 2 show marked discrepancies, which are undoubtedly associated with the different softness of the potential cores, whereas the isothermal compressibility of these systems are very similar.

\section{Transverse current correlations}

In order to analyze the propagation of shear modes we determined the transverse current correlation functions and the corresponding spectra, being the former defined as $[3,19]$

$$
C_{T}(k, t)=\frac{2}{N}\left\langle j_{\mathbf{k}}^{x}(t) \cdot j_{-\mathbf{k}}^{x}(0)\right\rangle
$$



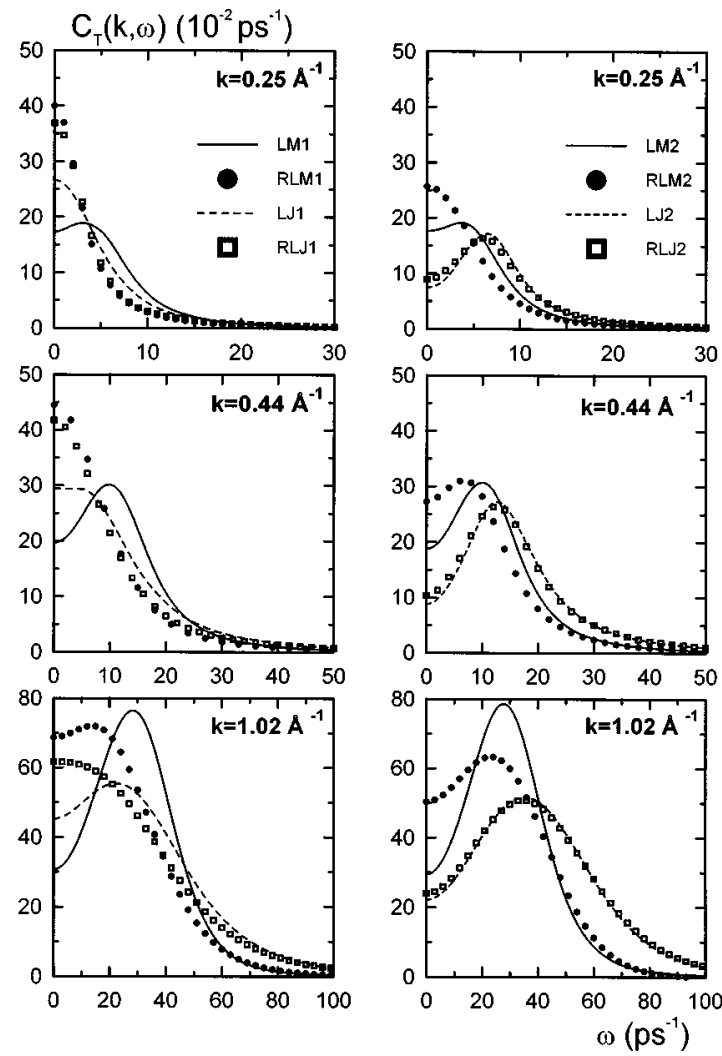

FIG. 8. Transverse current correlation functions at low $k$ values for the systems at the states 1 (left) and 2 (right).

where $j_{k}^{x}(t)$ is the part of the current function $\left[\vec{j}_{k}(t)\right]$ that is perpendicular to the direction of the wave vector $\vec{k}$. The most significant $C_{T}(k, \omega)$ results for the current transverse spectra $C_{T}(k, \omega)$ are shown in Fig. 8. The form of $C_{T}(k, \omega)$, for a given $k$, should be a Lorentzian at low $k$ 's (hydrodynamic regime) and a Gaussian at high $k$ 's (kinetic regime). However, it is well known that the $C_{T}(k, \omega)$ spectra of dense liquids can show a peak at nonzero frequency in the intermediate $k$ range. This peak, which reflects the propagation of shear waves in the liquid, is attributed to viscoelastic effects. We have obtained the maximum $\left(k_{T}^{\max }\right)$ and minimum $\left(k_{T}^{\min }\right)$ wave vectors of the $k$ interval at which a peak in $C_{T}(k, \omega)$ may be observed (as for $k_{L}^{\max }$, the values of $k_{T}^{\max }$ and $k_{T}^{\min }$ can only be approximately estimated). The $k_{T}^{\max }$ and $k_{T}^{\min }$ results (Table II) are strongly dependent on the thermodynamic state of the systems. The $C_{T}(k, \omega)$ functions for the systems at the state 2 show a peak for wider $k$ intervals than the ones for the systems at the state 1 whereas the $C_{T}(k, \omega)$ functions corresponding to the state 3 do not show any noticeable peak. According to these findings, liquids at low density and high temperature cannot sustain the propagation of shear waves. Moreover, the comparison of the $k_{T}^{\max }$ and $k_{T}^{\min }$ values for the full and the associated repulsive potentials prove that the attractive interatomic forces significantly favor the propagation of shear waves. This may be unambiguously observed from the $C_{T}(k, \omega)$ results for the systems at the state 1 whereas the results for the systems at the state 2 are less clear since the limits of the $k$ intervals for which the $C_{T}(k, \omega)$ 's show a peak are in several cases beyond the maximum and minimum values of $k$ considered in this study. Note that there is close agreement between the $C_{T}(k, \omega)$ functions for LJ2 and RLJ2 for all the analyzed $k$ 's while the functions for LM2 and RLM2 show noticeable differences, which should be attributed to the relatively strong attractive forces corresponding to LM2. The dependence of the propagation of shear modes on the softness of the potential cores is not so clear as for longitudinal modes. The $k_{T}^{\min }-k_{T}^{\max }$ interval is slightly wider for RLM1 than for RLJ1, which suggest that propagation of shear waves can be favored by potentials with soft cores. However, this is not corroborated by the results for state 2 since $k_{T}^{\min }$ for RLM2 is slightly higher than that for RLJ2 ( $k_{T}^{\max }$ for both systems is higher than the maximum value of $k$ considered in this study).

In the hydrodynamic regime [3,19], the $C_{T}(k, t)$ functions show the typical exponential decay of diffusive processes according to the equation $C_{T}(k, t)=\omega_{0}^{2} \exp \left(-\nu_{s} k^{2} t\right)$, where $\nu_{s} \equiv \eta_{s} / \rho m$ is the kinematic shear viscosity. An exponentiallike time decay of the $C_{T}(k, t)$ 's at low $k$ 's has been observed for the systems with the lowest viscosity (LJ3, RLJ3). However, the time decay of $C_{T}(k, t)$ for a fixed $k$ corresponding to the systems with the highest viscosity (LM1, LM2, LJ2, and RLJ2) is clearly nonexponential even for the lowest value of $k$ considered in this study (the $\eta_{s}$ coefficients are given in Table II). It should be noted that the decay of the $C_{T}(k, t)$ functions with a peak in their spectra must be nonexponential. The correlation between the $C_{T}(k, t)$ forms and the $\eta_{s}$ values corroborates that the propagation of shear modes is strongly associated with the viscoelastic effects.

\section{Transport coefficients}

The shear $\left(\eta_{s}\right)$ and longitudinal $\left(\eta_{l}\right)$ viscosity coefficients as well as the isothermal conductivity $(\lambda)$ have been calculated for the different systems in this paper by using both the Green-Kubo equations and the Einstein-like relations $[3,11,19]$. The transport coefficients obtained from the two procedures do not show significant discrepancies. A summary of the resulting coefficients is given in Table II. The values of the $\eta_{s}$ and $\eta_{l}$ coefficients for the RLM and RLJ potentials show that the viscosity is higher for the harder potential cores. Moreover, the influence of the softness of the potential core on the the viscosity coefficients is more marked at higher-packing fractions. The value of $\lambda$ also increases with the hardness of the potential core. The comparison between the results for the full and the corresponding repulsive potentials shows that the attractive interactions produce an increase of the three transport coefficients.

\section{CONCLUDING REMARKS}

The findings obtained in this paper corroborate that both the velocity autocorrelation functions and the dynamic structure factors are strongly dependent on the softness of the potential cores. According to earlier results for soft-sphere fluids [18], the Brillouin peaks in the $S(k, \omega)$ 's are more remarkable and persist up to larger wave numbers for the softer potentials. Our results suggest that soft core interactions allow the coherence necessary for the collective motions of the atoms and the propagation of longitudinal modes. However, the resulting $C_{T}(k, \omega)$ 's show that the propagation of shear modes is not very much influenced by 
the softness of the potential cores. These results are consistent with the differences in the range of wavelength over which longitudinal and transverse modes propagate in raregas liquids and liquid-alkali metals [3,5]. Moreover, they confirm that the different dynamic behavior of LM and LJ liquids should be mainly attributed to the different softness of the potential cores.

Besides, our findings also show that the influence of the attractive interactions on the dynamic properties can be important in some thermodynamic states. So, we have observed that the different properties $\left[C(t), S(k, \omega)\right.$, and $\left.C_{T}(k, \omega)\right]$ of systems at low packing fractions are significantly dependent on the effects of the attractive forces. For the same thermo- dynamic conditions, these effects are more marked for LM than for LJ potentials, as corresponds to the weaker attractions of the second. At high packing fractions, the effects of the attractive forces are screened out and the propagation of longitudinal and shear modes is basically due to the almost continuous collisions among the close atomic cores.

\section{ACKNOWLEDGMENTS}

We thank N. Anento for his valuable contribution to the final version of this paper. Financial support from DGICYT (Grant No. PB96-0170-C03) and CIRIT (Grant No. 1997SGR-00149) is gratefully acknowledged.
[1] N. H. March, Liquid Metals (Cambridge University, Cambridge, 1990).

[2] A. Rahman, Phys. Rev. Lett. 32, 52 (1974).

[3] J. P. Hansen and I. R. McDonald, Theory of Simple Liquids (Academic, London, 1986).

[4] D. Schiff, Phys. Rev. 186, 151 (1969).

[5] G. Jacucci and I. R. McDonald, Mol. Phys. 39, 515 (1980).

[6] J. W. E. Lewis and S. W. Lovesey, J. Phys. C 10, 3221 (1977).

[7] I. Ebbsjo, T. Kinell, and I. Waller, J. Phys. C 13, 1865 (1980).

[8] M. Canales and J. A. Padró, Phys. Rev. E 56, 1759 (1997).

[9] G. Kahl and S. Kambayashi, J. Phys.: Condens. Matter 6, 10897 (1994).

[10] L. E. González, D. J. González, M. Silbert, and J. A. Alonso, J. Phys.: Condens. Matter 5, 4283 (1993); M. Canales, J. A. Padró, L. E. González, and A. Giró, ibid. 5, 3095 (1993).

[11] M. Canales, L. E. González, and J. A. Padró, Phys. Rev. E 50, 3656 (1994).

[12] M. Canales and J. A. Padró, J. Phys.: Condens. Matter 9, 11009 (1997).

[13] R. W. Zwanzig, J. Chem. Phys. 22, 1420 (1954); B. Widom, Science 157, 375 (1967); D. Henderson and J. A. Barker, J. Chem. Phys. 52, 2315 (1970).
[14] J. D. Weeks, D. Chandler, and H. C. Andersen, J. Chem. Phys. 54, 5237 (1971); H. C. Andersen, D. Chandler, and J. D. Weeks, Adv. Chem. Phys. 34, 105 (1976).

[15] D. Chandler, J. D. Weeks, and H. C. Andersen, Science 220, 787 (1983); J. D. Weeks, R. L. B. Selinger, and J. Q. Broughton, Phys. Rev. Lett. 75, 2694 (1995).

[16] A. Torcini, U. Balucani, P. H. K. de Jong, and P. Verkerk, Phys. Rev. E 51, 3126 (1995); G. Nowotny, G. Khal, and J. Hafner, Phys. Scr. 57, 22 (1995); S. Kambayashi, G. Nowotny, J. Chihara, and G. Khal, J. Non-Cryst. Solids 205-207, 914 (1996).

[17] U. Balucani, A. Torcini, and R. Vallauri, Phys. Rev. B 47, 3011 (1993).

[18] S. Kambayashi and Y. Hiwatari, Phys. Rev. E 49, 1251 (1994).

[19] U. Balucani and M. Zoppi, Dynamics of the Liquid State (Clarendon, Oxford, 1994).

[20] W. E. Alley, B. J. Alder, and S. Yip, Phys. Rev. A 27, 3174 (1983).

[21] M. M. G. Alemany, C. Rey, and L. J. Gallego, J. Chem. Phys. 105, 8250 (1996). 